\title{
Low temperature growth of vertically aligned carbon nanotubes via floating catalyst chemical vapor deposition method.
}

\begin{abstract}
Synthesis of carbon nanotubes (CNTs) below $600^{\circ} \mathrm{C}$ using supporting catalyst chemical vapor deposition method was reported by many research groups. However, the floating catalyst chemical vapor deposition received less attention due to imperfect nanotubes produced. In this work, the effects of varying the preheating temperature on the synthesis of CNT were investigated. The reaction temperature was set at $570^{\circ} \mathrm{C}$. The preheating set temperature was varied from 150 to $400^{\circ} \mathrm{C}$ at $50^{\circ} \mathrm{C}$ interval. Three O-ring shape heating mantels were used as heating source for the preheater. In situ monitoring device was used to observe the temperature profile in the reactor. Benzene and ferrocene were used as the carbon source and catalyst precursor, respectively. Vertically aligned CNTs were synthesized when the preheating temperature was set at $400^{\circ} \mathrm{C}$. When the preheating temperature was increased up to $400^{\circ} \mathrm{C}$, both the length and the alignment of CNTs produced were improved.
\end{abstract}

Keyword: Vertically aligned carbon nanotubes (CNTs); Floating catalyst; Low synthesis temperature; Preheating set temperature. 